\title{
STRUCTURAL RELIABILITY AND ITS SENSITIVITY ANALYSIS BASED ON THE SADDLEPOINT APPROXIMATION-LINE SAMPLING METHOD BY DICHOTOMY OF GOLDEN SECTION
}

\author{
Ganqing Zhang * - Yanghui Xiang - Huixin Guo - Lei Guo - Yonghong Nie
}

Department of Mechanical \& Electrical Engineering, Changsha University, Changsha, China,410003

\begin{tabular}{l} 
ARTICLE INFO \\
\hline Article history: \\
Received: 28.06 .2016$. \\
Received in revised form: 12.12.2016. \\
Accepted: 21.12 .2016$. \\
\hline Keywords: \\
Saddlepoint approximation (SA) \\
Line sampling (LS) \\
Golden section method \\
Dichotomy \\
Reliability sensitivity \\
\hline DOI: http://doi.org/10.30765/er.39.1.2
\end{tabular}

\section{Introduction}

In practicable engineering, when the traditional Monte Carlo simulation (MSC) is used to solve the structural reliability and its sensitivity with the nonnormal random variables, for small probabilities, its sampling efficiency is so low that it is difficult to be accept by the engineers because of its calculation amount. For the first/second order reliability

\begin{abstract}
:
In order to solve the structural reliability and its sensitivity of the implicit nonlinear performance function (PF) the advantages of the saddlepoint approximation (SA) and line sampling (LS) are merged. Also, the merits of dichotomy and the solution efficiency of the golden section method are combined to propose the saddlepoint approximation-line sampling (SA-LS) method based on the dichotomy of the golden section point. This is complicated and changeable in the non normal variable space, which is a very hot issue of the present international study. For each sample, it is quick to find its zeropoint in PF along the important line sampling direction by the previously mentioned dichotomy so that the structural failure probability can be transformed into the mean of a series linear PFs failure probability, and the reliability sensitivity is just the derivative or partial one of the probabilities with respect to the relational variables. Examples show that the SA-LS method based on the dichotomy of the golden section point is of high precision and fast velocity in analyzing the structural reliability and sensitivity of the implicit nonlinear PF that are complicated and changeable in the non-normal variable space.
\end{abstract}

method, they can only analyze the structural reliability and its sensitivity when the non-normal variables are transformed into the standard normal variables. This is a nonlinear course in such a way that it will increase the nonlinear degree of the performance function (PF) and decrease the precision of reliability analysis [1,2], which also restrict their application. The conventional line sampling (LS) method [3] can estimate with high efficiency the reliability and its sensitivity of the

\footnotetext{
${ }^{*}$ Corresponding author. Tel.: +86-13667388819

E-mail address: hnyyzgq@163.com
} 
high-dimensional and small probabilities by the randomly sampling in $n-1-\mathrm{D}$ space and 1-D interpolation and transforming the failure probability (FP) of the nonlinear PF into the FP arithmetic mean of a series linear PF. However, the whole LS course is completed in the standard normal space. Its precision will be unavoidably affected by the nonlinearly standardized transformation. Moreover, the error is obtained once the PF appears with non-monotonic tendency because of the transformation. The saddlepoint approximation (SA) [4-7] adopts the cumulative generating function (CGF) (or moment generating function, MGF) of the random variables to estimate the cumulative distribution function (CDF) or FP of the structural response PF. It isn't restricted by the basic variable distribution type so that it is suitable to non-normal variables. However, it demands that the PF must be linear. To cater to the need, documents [8-10] expand the nonlinear PF into approximate linear expressions in mean and design points according to the Taylor formula. The process mode will however difficultly obtain the approximate solution to meet the precision needed for those problems whose nonlinear degree is high or whose variance coefficient of the random variable is very big instead of its high nonlinear degree. What's more, it still demands outputting the gradient function of PF, and it isn't easy for the implicit PF. Using the advantages that SA doesn't make demand of the basic variable distribution type and that LS is adaptive for the nonlinear PF. Document [7] proposes the SA-LS method and smoothly merges their complementary advantages and effectively solves the reliability of the one-peak $\mathrm{PF}$, for those PFs that are complicated and changeable, especially for those PFs that there are multi-peaks in LS direction. However, it is helpless for the 3-points quadratic interpolation ${ }^{[12,7]}$ to answer the zeropoint of PF and the saddlepoint of CGF differential equation. Therefore, document [13] uses linear interpolation method to solve it, but it still needs to get the gradient functions of PF so that it is unsuitable for the implicit functions in practicable engineering that are obtained by the approximate method. Because the dichotomy shouldn't output beforehand the gradient function of PF. It is not only suitable for the implicit PF, but also for the multi-peaks PF, referring to the solution efficiency of the gold section. The paper puts forward the SA-LS method based on the dichotomy of the gold section to further solve the reliability and its sensitivity of the implicit PFs from practicable engineering that are complicated and changeable.

\section{The reliability and its sensitivity analysis based on the SA}

\subsection{The reliability analysis based on the SA}

The basic idea of the SA is to use the CGF properties of linear $\mathrm{PF}$ with the random variable and the inverse Fourier transform (IFT) to get the exponential power series expressions of $\mathrm{PF}$ probability density function (PDF) based on $\mathrm{SA}^{[4,13]}$. Suppose $f(x)$ is the PDF of the basic random variables $x$, and its moment generating function (MGF) is

$$
M_{x}(t)=\int_{-\infty}^{+\infty} e^{t x} f(x) d x
$$

Then its CGF is

$$
K_{x}(t)=\ln \left[M_{x}(t)\right]
$$

The CGFs of the common distribution see the literature 6 , they have the properties as following (1)if $x_{1}, x_{2}, \cdots, x_{n}$ are $n$-D mutually independent basic variables, and their CGF are $k_{x_{i}}(t)(i=1,2, \cdots, n)$, respectively, then the CGF of the response PF $y=\sum_{i=1}^{n} x_{i}$ is

$$
K_{y}(t)=\sum_{i=1}^{n} k_{x_{i}}(t)
$$

(2)Suppose the CGF of the mutually independent basic random variable $x$ is $K_{x}(t)$, and the CGF of linear response PF $y=a x+b$ is

$$
K_{y}(t)=K_{x}(a t)+b t
$$

Therefore, for the mutually independent basic variables $x_{1}, x_{2}, \cdots, x_{n}$, if their linear $\mathrm{PF}$ is $y=a_{0}+\sum_{i=1}^{n} a_{i} x_{i}$, then CGF of $y$ is 


$$
K_{y}(t)=a_{0} t+\sum_{i=1}^{n} K_{x_{i}}\left(a_{i} t\right)
$$

By using the IFT, the PDF $f_{Y}(y)$ of $y$ can be represented by

$$
\begin{aligned}
f_{Y}(y) & =\frac{1}{2 \pi} \int_{-\infty}^{+\infty} M(i \xi) e^{-i \xi y} d \xi \\
& =\frac{1}{2 \pi} \int_{-\infty}^{+\infty} \exp [K(t)-\xi y] d \xi
\end{aligned}
$$

When using the expanded formula of the exponential power series to estimate Eq.(6), Daniels $^{[4]}$ obtained the saddlepoint expression of $f_{Y}(y)$ as following

$$
f_{Y}(y) \approx\left[\frac{1}{2 \pi K_{y}^{\prime \prime}\left(t_{s}\right)}\right]^{\frac{1}{2}} e^{\left[K_{y}\left(t_{s}\right)-t_{s} y\right]}
$$

Here, $K_{y}^{\prime \prime}(\cdot)$ is the $2^{\text {nd }}$ order derivative of $K_{y}(t)$ with respect to $t$.The saddlepoint $t_{s}$ is the solution of the nonlinear equation $K_{y}^{\prime}(t)=y_{0}$, where $y_{0}$ is the preset threshold value.

According to the SA, the $\operatorname{CDF} F_{y}\left(y_{0}\right)$ of $y$ is

$$
F_{y}\left(y_{0}\right)=P\left\{y \leq y_{0}\right\} \approx \Phi\left(w+\frac{1}{w} \ln \left(\frac{w}{v}\right)\right)
$$

Here

$$
\begin{gathered}
w=\operatorname{sgn}\left(t_{s}\right)\left\{2\left[t_{s} y_{0}-K_{y}\left(t_{s}\right)\right]\right\}^{\frac{1}{2}} \\
v=t_{s}\left[K_{y}^{\prime \prime}\left(t_{s}\right)\right]^{\frac{1}{2}}
\end{gathered}
$$

Where, the values of the symbolic function $\operatorname{sgn}\left(t_{s}\right)$ are 1,0 and -1 , which are corresponding to $t_{s}>0, t_{s}=0$ and $t_{s}<0$, respectively.

Utilizing the CDF expressions of the response PF $y$ in Eq.(8), its FP $P_{f}$ can be resulted from $F_{y}(0)$ corresponding to $y<0$, namely, $w+1 / w \cdot \ln (w / v)$ in Eq.(8) is regarded as the inverse number of the probability index $\beta$ in the equivalent normal variable space, thus

$$
P_{f}=\Phi(-\beta)=\Phi\left(w+\frac{1}{w} \ln \left(\frac{w}{v}\right)\right)
$$

\subsection{The reliability sensitivity analysis based on the SA}

As can be seen from section 2.1, the SA can express the FP of the PF with non-normal variables as the form of standard normal CDF, therefore, for the partial derivative of Eq.(11) with respect to $\theta$, the reliability sensitivity based on the SA is written as

$$
\begin{aligned}
\frac{\partial P_{f}}{\partial \theta} & =-\frac{1}{\sqrt{2 \pi}} \exp \left(-\frac{\beta^{2}}{2}\right) \cdot \beta \cdot \frac{\partial \beta}{\partial \theta} \\
& =-\frac{1}{\sqrt{2 \pi}} \exp \left(-\frac{\beta^{2}}{2}\right) \cdot \beta \cdot\left(\frac{\partial \beta}{\partial w} \frac{\partial w}{\partial \theta}+\frac{\partial \beta}{\partial \nu} \frac{\partial v}{\partial \theta}\right)
\end{aligned}
$$

Here

$$
\begin{gathered}
\frac{\partial \beta}{\partial w}=-\left[1+\frac{1}{w^{2}}\left(1-\ln \left(\frac{w}{v}\right)\right)\right] \\
\frac{\partial \beta}{\partial v}=\frac{1}{v w}
\end{gathered}
$$

Utilizing the function relationship between the CGF and its $2^{\text {nd }}$ order derivative and distribution parameter $\theta, \partial w / \partial \theta$ and $\partial v / \partial \theta$ can be derived from Eq.(9) (10) so that $\partial P_{f} / \partial \theta$ can be drawn out.

It is noteworthy that in the course of deriving the CDF of PF $y$ in Eq.(8),it isn't essential setting beforehand the distribution forms of variable $x_{i}$, so it is suitable for any variable. It, however, demands $y$ is the linear function of $x_{i}$, while the practicable PF $y=g(x)$ is often nonlinear, therefore, the method as following is proposed specially.

\section{The reliability and its sensitivity analysis based on the SA-LS method by the dichotomy of the golden section}

To avoid deriving of the nonlinear PF when it is linearized, and to answer the reliability and its sensitivity of the PFs that are sophisticated and changeable, especially for the PFs that have multipeaks in LS direction, the paper uses the advantages of the SA which are not being restricted by the basic 
variable distribution type and of the LS which are not suitable for nonlinear PFs. It also merges the merits of dichotomy and the efficiency of the gold section to propose the SA-LS based on the dichotomy of the gold section. Its basic idea is that the non-normal variable is first standardized, and that the reliability and its sensitivity is translated into the mean of reliability and its sensitivity of a series linear PF according to the probability distribution in the standardized space. Furtheremore, the dichotomy of the gold section is used to solve the zeropoint of PF along the LS direction and the saddlepoint of the CGF differential equation. Finally, the SA is used to solve the FP and the sensitivity of FP with respect to distribution parameters of the linear PF in nonnormal space. The concrete steps are as follows.

\subsection{Standardizing variable space and its important direction}

Let $x=\left(x_{1}, x_{2}, \cdots, x_{n}\right)^{T}$, if $\mu_{x_{i}}$ and $\sigma_{x_{i}}$ are the mean and variance, respectively, of the basic variable $x_{i}$, and it is linearly standardized as follows to get rid of the dimension's effect.

$$
z_{i}=\frac{x_{i}-\mu_{x_{i}}}{\sigma_{x_{i}}} \quad i=1,2, \cdots, n
$$

In the standardized space $z$ defined as Eq.(15), $\bar{g}(z) \leq 0$ defines the failure field $F$, if the maximum point of the joint PDF value is treated as the design point $Z^{*}$, then the vector from the origin pointing to the design point $z^{*}$ is just important direction $\alpha$.Its unit vector $e_{\alpha}=\alpha /\|\alpha\|$ is obtained by regularizing $\alpha$.

\subsection{The dichotomy of the gold section in important direction}

For any vector $z$ in the standardized variable space, it is decomposed into the adding form of the vector $c e_{\alpha}$ and $z^{\perp}$. The former is the vector that is parallel to important direction, the latter is the vector that is vertical to the direction, that is

$$
z=c e_{\alpha}+z^{\perp}
$$

Here, $c=<e_{\alpha}, z>,\left\langle e_{\alpha}, z>\right.$ is the dot product of vector $e_{\alpha}$ and $z$.

Eq.(16) can be rewritten as $z^{\perp}=z-<e_{\alpha}, z>e_{\alpha}$,vector $z^{\perp}$ can thus be obtained which is corresponding to vector $z$ and vertical to the important direction $e_{\alpha}$.

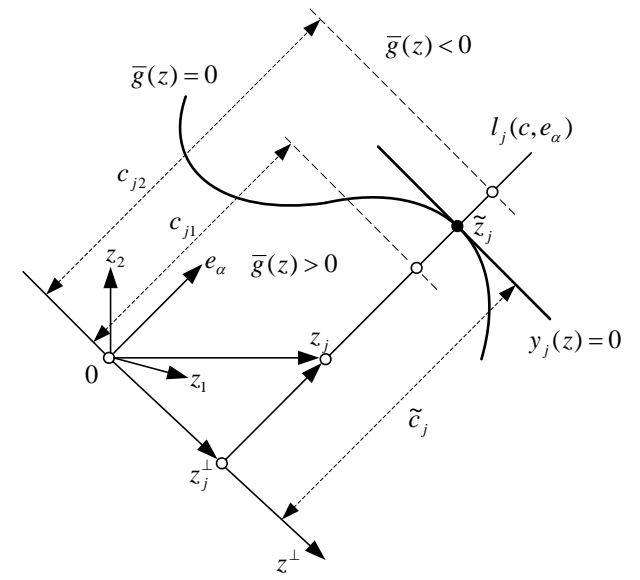

Figure 1. The SA-LS method based on the dichotomy of the golden section point.

After $N$ samples $x_{j}(j=1,2, \cdots, N)$ are produced in accordance with the PDF $f_{x}(x)$ of $x$, they are standardized as $z_{j}(j=1,2, \cdots, N)$ according to Eq.(15), then the vector $z_{j}^{\perp}=z_{j}-<e_{\alpha}, z_{j}>e_{\alpha}$ is obtained which is vertical to $e_{\alpha}$ according to the method above, as is shown in Fig. 1. According to the idea of dichotomy, for any vector $z_{j}$, once 2 coefficients $c_{j 1}$ and $c_{j 2}$ are given along the line $l_{j}\left(c, e_{\alpha}\right)$,their corresponding vector $c_{j i} e_{\alpha}+z_{j}^{\perp}$ and the PF $\bar{g}\left(c_{j i} e_{\alpha}+z_{j}^{\perp}\right)(i=1,2)$ are obtained (it is demanded that the given 2 coefficients must make their $\mathrm{PF}$ values opposite sign). Then we can answer the gold section point $c_{j 3}=c_{j 1}+(\sqrt{5}-1) \times\left(c_{j 2}-c_{j 1}\right) / 2$ and its vector $c_{j 3} e_{\alpha}+z_{j}^{\perp}$ and the corresponding $\mathrm{PF}$ $\bar{g}\left(c_{j 3} e_{\alpha}+z_{j}^{\perp}\right)$.The operation is circulated by the idea of the dichotomy until the preset accuracy is met. If the obtained final intersection point of the line $l_{j}\left(c, e_{\alpha}\right)$ and the limited state function (LSF) $\bar{g}(z)=0$ is $\tilde{z}_{j}$ and its corresponding multiple is $\widetilde{c}_{j}$, then 


$$
\tilde{z}_{j}=\widetilde{c}_{j} e_{\alpha}+z_{j}^{\perp}
$$

Noting, when the dichotomy of the golden section is used to answer the zeropoint $\widetilde{z}_{j}$ along the LS direction, for any sample $z_{j}$, the points $z_{j 1}$ and $z_{j 2}$ which are corresponding to the selected 2 coefficients $C_{j 1}$ and $c_{j 2}$ along the line direction of the unit vector $e_{\alpha}$ must make their PF values' opposite sign. This is because they may locate in the direction of $e_{\alpha}$, and maybe go in its opposite direction, so it is easier to find 2 points which meet the requirement when we use the command randn instead of the command rand. What's more, there is no need in considering its direction.

\subsection{The linear transformation of nonlinear PF for the FP and its sensitivity}

If $y_{j}(z)=0$ is the hyperplane that passes the intersection $\tilde{z}_{j}$ and that is vertical to the unit important direction $e_{\alpha}$, then it is determined by Eq.(18)

$$
y_{j}(z)=-e_{\alpha}\left(z-\tilde{z}_{j}\right)=-\sum_{i=1}^{n} e_{\alpha i}\left(z_{i}-\tilde{z}_{j i}\right)
$$

Here, $e_{\alpha i}$ and $\tilde{z}_{j i}$ are the $i$ th components of $e_{\alpha}$ and $\tilde{z}_{j}$, respectively.

According to the basic principle of the LS method, the FP corresponding to $\bar{g}\left(\tilde{z}_{j}\right) \leq 0$ can be estimated by Eq.(19)

$$
\hat{P}_{f}=\frac{1}{N} \sum_{j=1}^{N} P_{f j}
$$

Here, $P_{f j}=P\left\{y_{j}\left(\tilde{z}_{j}\right) \leq 0\right\}$ is the FP of the failure region of the linear PF $y_{j}\left(\tilde{z}_{j}\right)$.

Therefore, the estimated value of reliability sensitivity is

$$
\frac{\partial \hat{P}_{f}}{\partial \theta}=\frac{1}{N} \sum_{j=1}^{N} \frac{\partial P_{f j}}{\partial \theta}
$$

Therefore, for the saddlepoint $t_{s}$ of the nonlinear equation $K_{y}^{\prime}(t)=y_{0}$ in section 2 , when using the proposed dichotomy of the golden section in section 3.2 , we can be sure to get a closed solution so that the problem of the finite difference can be avoided effectively. It is worth mentioning that, for the Gumbel distribution, the absolute value must be added in the solving process to meet the domain when we use the command randn. Similarly, for the uniform distribution, it also requires $t \neq 0$, otherwise it will be stuck.

\subsection{The SA of linear PF for the FP and its sensitivity}

According to the linearization transformation from $x$ to $z$, Eq. (18) is translated into the original variable space, then

$$
\begin{aligned}
y_{j} & =-\sum_{i=1}^{n} e_{\alpha i}\left(z_{i}-\tilde{z}_{j i}\right)=-\sum_{i=1}^{n} e_{\alpha i}\left(\frac{x_{i}-\tilde{x}_{j i}}{\sigma_{x_{i}}}\right) \\
& =\sum_{i=1}^{n} e_{\alpha i} \frac{\tilde{x}_{j i}}{\sigma_{x_{i}}}+\sum_{i=1}^{n}\left(-e_{\alpha i} \frac{x_{i}}{\sigma_{x_{i}}}\right)
\end{aligned}
$$

For the linear PF $y_{j}$ above, it is inferred from the SA in section 2.1 that its CGF is

$$
K_{y_{j}}(t)=\sum_{i=1}^{n} e_{\alpha i} \frac{\tilde{x}_{j i}}{\sigma_{x_{i}}} t+\sum_{i=1}^{n} K_{x_{i}}\left(-\frac{e_{\alpha i}}{\sigma_{x_{i}}} t\right)
$$

For every failure point of linear PF of Eq.(18), we can draw out its FP $P_{f j}=P\left\{y_{j} \leq 0\right\}$ and the partial derivative with respect to the distribution parameter $\partial P_{f j} / \partial \theta$ according to the $\mathrm{SA}$, once they are substituted into Eq.(19) (20), the estimation value of the structural $P_{f}$ and $\partial P_{f} / \partial \theta$ can be obtained.

3.5 The realization of the reliability and its sensitivity analysis based on the SA-LS method by the dichotomy of the golden section

To make the proposed algorithm understood and operated easily, the course of its realization is generalized in Fig. 2. The concrete computable steps are as following: (1) Standardizing the space 
variables according to their mean values and standard deviations, respectively. (2) Finding and unitizing the important direction. (3) Describing the vectors in the important direction.

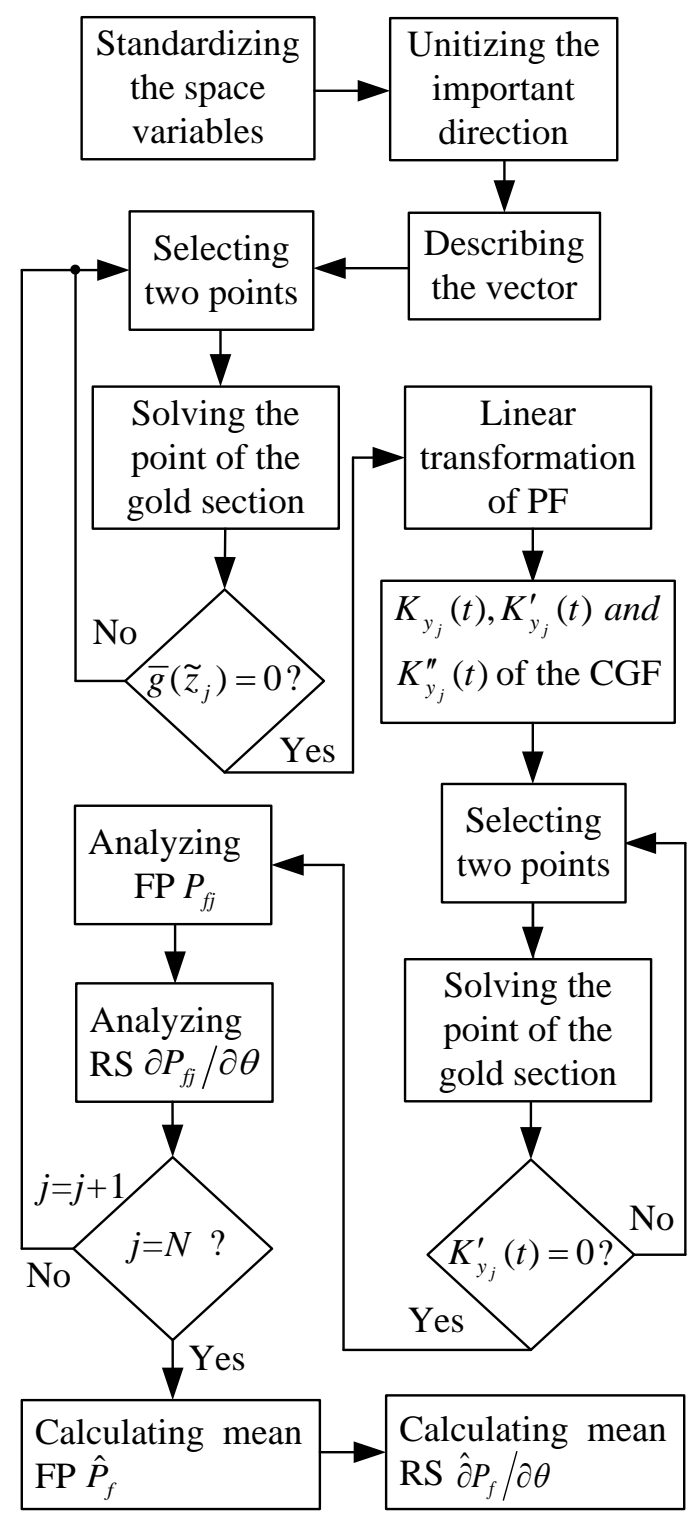

Figure 2. Flow chart of reliability \& its sensitivity by the SA-LS method of the gold section dichotomy.

(4) Selecting two points that meet the demands along the pointed direction, finding the point of the golden section, and computing its PF value. For the point of the golden section $\widetilde{z}_{j}$, judging if $\bar{g}\left(\tilde{z}_{j}\right)$ is equal to 0 , if $\bar{g}\left(\tilde{z}_{j}\right) \neq 0$, then looking back for the next golden section point. (5) If $\bar{g}\left(\tilde{z}_{j}\right)=0$, then the nonlinear PF is transformed into the linear one. (6)
The CGF $K_{y_{j}}(t)$ and its first and second order differential functions $K_{y_{j}}^{\prime}(t)$ and $K_{y_{j}}^{\prime \prime}(t)$ are derived out. (7) The dichotomy of the golden section is used to solve the saddlepoint $t_{s}$ which is the solution of the equation $K_{y_{j}}^{\prime}(t)=0$ once more.(8) If $K_{y_{j}}^{\prime}(t)=0$, then the FP $P_{f j}$ and the reliability sensitivity (RS) $\partial P_{f j} / \partial \theta$ are analyzed.(9) If $j \neq N$, let $j \neq j+1$, return back to step (4), If $j=N$, the mean FP $\hat{P}_{f}$ and RS $\hat{\partial} P_{f} / \partial \theta$ can be calculated out.

\section{Illustrative examples}

The gearbox of the 3-stage planetary gear reducer (PGR) from the Earth-Press-Balance shield machine serves as an example to check the effectiveness of the proposed algorithm. For the PGR, its input torque $T_{s}$ is $1489 \mathrm{~N} . \mathrm{m}$, the materials of its gearbox is $17 \mathrm{CrNi2MoAl}$, whose yield strength $\sigma_{s}$ is $785 \mathrm{Mpa}$. Suppose the basic variables are mutually independent, the basic parameters involved in design course are shown in Table 1 and Table 2.

Table 1. The basic parameter values of each-stage PGR

\begin{tabular}{|l|c|c|c|}
\hline name & $\begin{array}{l}1^{\text {st }} \\
\text { stage }\end{array}$ & $\begin{array}{l}2^{\text {nd }} \\
\text { stage }\end{array}$ & $\begin{array}{l}3^{\text {rd }} \\
\text { stage }\end{array}$ \\
\hline $\begin{array}{l}\text { Gear number of } \\
\text { sun gear } z_{s}\end{array}$ & 21 & 25 & 24 \\
\hline $\begin{array}{l}\text { Gear number of } \\
\text { internal gear } z_{r}\end{array}$ & 55 & 63 & 76 \\
\hline $\begin{array}{l}\text { the number of } \\
\text { planetary gear } n_{p}\end{array}$ & 4 & 4 & 5 \\
\hline $\begin{array}{l}\text { the length of } \\
\text { gearbox } b / \mathrm{mm}\end{array}$ & 57 & 92 & 114 \\
\hline
\end{tabular}

When the bending moment is ignored resulting from the gearbox self-weight, the gearbox stress exists in two sources as following, (1) the bending stress resulting from the torque of the gear train acting on the gear teeth of internal gear, (2) the pressure stress resulting from the pre-tightening force of tightening bolt. According to the analysis results of the finite element method, the later is far smaller than the former, so the stress from the tightening bolt is neglected in the course of building mechanical 
model of the gearbox in this paper. Therefore, the torque acting on the single gear can be represented by

$$
T=\frac{T_{r}}{\varepsilon n_{p}}, \quad T_{r}=p T_{s}, \quad p=\frac{Z_{r}}{Z_{s}}
$$

Here, $T$ is the torque acting on the single gear, $T_{r}$ and $T_{s}$ are the torque of the internal and sun gear, respectively. $z_{s}$ and $z_{r}$ are the gear number of the sun and internal gear, respectively. $n_{p}$ is the number of the planetary gear. $p$ is the characteristic parameter of the planetary row. $\varepsilon$ is the coefficient of the overlap ratio.

For the PGR whose type is $2 \mathrm{Z}-\mathrm{X}\left(i^{x}<0\right)$, eachstage input torque $T_{s_{i}}(i=1,2,3)$ existing in the relation as following $[15,16]$.

$$
T_{s_{i}}=\left(1+p_{i-1}\right) T_{s_{i-1}} \quad(i=2,3)
$$

Obviously, the torque $T$ imposed on the gearbox mainly acts on the neighborhood of single gear. Let $M=T$, if there is a screw hole in the region, as is shown in Fig. 3, because its cross-section coefficient is the smallest, so section $a a$ is just the most dangerous one. Therefore, when the center of screw hole lies in the midpoint of gearbox thickness, the anti-bending cross-section coefficient is

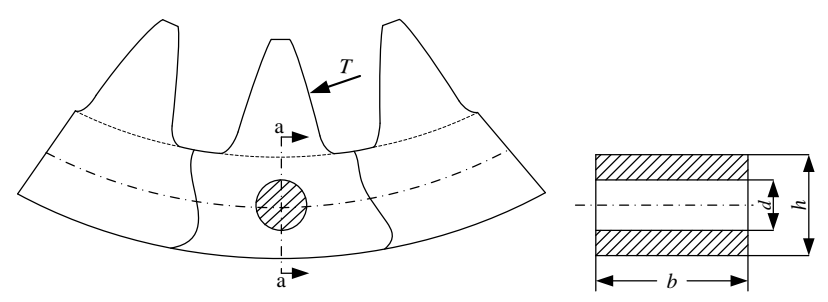

Figure 3. Force figure of the gearbox.

$$
W=\left(\frac{1}{12} b h^{3}-\frac{1}{12} b d^{3}\right) / \frac{h}{2}=\frac{b}{6 h}\left(h^{3}-d^{3}\right)
$$

Here, $b$ and $h$ are the length and thickness of the gearbox, respectively, $d$ is the radius of the screw hole.
So the bending stress of the cross-section is

$$
\sigma^{\prime}=\frac{M}{W}=\frac{6 h T_{s_{i}}}{\varepsilon b\left(h^{3}-d^{3}\right)} \cdot \frac{z_{r}}{z_{s} n_{p}}
$$

Referring to the idea of computing the bending stress of tooth root ${ }^{[16]}$, the coefficient of the overlap ratio $Y_{\text {srp }}$, the modified coefficient of stress $Y_{S a}$, the coefficient of tooth profile $Y_{\mathrm{Fa}}$ and the coefficient of load $K$ are introduced to correct the practicable engagement stress of tooth root in section $a a$ of gearbox, and there is

$$
\sigma=Y_{\text {srp }} Y_{\mathrm{Sa}} Y_{\mathrm{Fa}} K \sigma_{\max }^{\prime}=K_{s} \sigma^{\prime}
$$

Here, $K_{s}=Y_{\text {srp }} Y_{S a} Y_{\mathrm{Fa}} K$,the values of each-stage coefficient are shown in Table 3.

For the most dangerous section $a a$, according to the theory of stress-strength interference, under the condition of considering certain safety margin, the LSF that is represented by the limited state stress is

$$
g(x)=\frac{\sigma_{s}}{S}-\sigma=\frac{\sigma_{s}}{S}-\frac{6 h T_{s_{i}} k_{s}}{\varepsilon b\left(h^{3}-d^{3}\right)} \cdot \frac{z_{r}}{z_{s} n_{p}}
$$

Here, $\sigma_{s}$ is the yield strength of the materials $x=\left[\sigma_{s}, S, T_{s_{i}}, k_{s}, \varepsilon, b, d, h\right]^{T}$.

The values of $z_{r}, z_{s}$ and $n_{p}$ are integer, and the engagement course is subject to strict constraints so that they can be treated as the common number, the basic random variable $x$ in Eq.(28), therefore, don't contain $z_{r}, z_{s}$ and $n_{p}$.It is seen from Eq.(28), that the PF $g(x)$ is nonlinear. The distribution type and eigenvalue of all basic parameters are shown in Table 2. Simultaneously, the distribution parameter values are calculated according to the basic type and eigenvalue of the variable in Table 2, and their results are also filled in Table 2, where the deviation $\sigma_{x}$ is computed by the given mean value $u_{x}$ multiplying the given variance coefficient $C_{V}$.

In Table 2, it is noted that for the uniform distribution, distribution parameter 1 and 2 are the lower and upper bound, respectively. However, for the distribution of the extreme value type I, lognormal distribution and normal distribution, distribution parameter 1 and 2 are the mean and 
variance, respectively. Their values of distribution parameters are different from those of the corresponding Eigen parameters but normal distribution.

Table 2. Distribution type and parameters values of the basic random variables

\begin{tabular}{|c|c|c|c|c|c|c|c|}
\hline \multicolumn{2}{|c|}{$x$} & $\begin{array}{l}\text { distribution } \\
\text { parameter } 1\end{array}$ & $\begin{array}{l}\text { distribution } \\
\text { parameter } 2\end{array}$ & $u_{x}$ & $\sigma_{x}$ & $\begin{array}{c}\text { variance } \\
\text { coefficient } \\
C_{V} \\
\end{array}$ & $\begin{array}{l}\text { Distribution } \\
\text { type }\end{array}$ \\
\hline \multicolumn{2}{|c|}{$\sigma_{s} / \mathrm{MPa}$} & 513.0680 & 1056.9320 & 785 & 157 & 0.2 & uniform \\
\hline \multirow{3}{*}{$S$} & $1^{\text {st }}$ stage & 1.9016 & 2.6984 & 2.3 & 0.23 & 0.1 & \multirow{3}{*}{ uniform } \\
\hline & $2^{\text {nd }}$ stage & 1.8603 & 2.6397 & 2.25 & 0.225 & 0.1 & \\
\hline & $3^{\text {rd }}$ stage & 1.6539 & 2.3494 & 2 & 0.2 & 0.1 & \\
\hline \multirow{3}{*}{$T_{s} / \mathrm{N} . \mathrm{m}$} & $1^{\text {st }}$ stage & 1354.9742 & 232.1937 & 1489 & 297.8 & 0.2 & \multirow{3}{*}{$\begin{array}{l}\text { extreme value } \\
\text { type I }\end{array}$} \\
\hline & $2^{\text {nd }}$ stage & 4903.9327 & 840.3572 & 5389 & 1077.8 & 0.2 & \\
\hline & $3^{\text {rd }}$ stage & 17261.5881 & 2958.0137 & 18969 & 3793.8 & 0.2 & \\
\hline \multirow{3}{*}{$k_{s}$} & $1^{\text {st }}$ stage & 1.8335 & 0.1760 & 6.3536 & 1.2707 & 0.2 & \multirow{3}{*}{ log-normal } \\
\hline & $2^{\text {nd }}$ stage & 1.1854 & 0.2402 & 3.3677 & 0.6735 & 0.2 & \\
\hline & $3^{\text {rd }}$ stage & 0.6138 & 0.3132 & 1.9404 & 0.3881 & 0.2 & \\
\hline \multirow{3}{*}{$\varepsilon$} & $1^{\text {st }}$ stage & 1.5723 & 0.0314 & 1.5723 & 0.0314 & 0.02 & \multirow{3}{*}{ normal } \\
\hline & $2^{\text {nd }}$ stage & 1.5077 & 0.0302 & 1.5077 & 0.0302 & 0.02 & \\
\hline & $3^{\text {rd }}$ stage & 1.4855 & 0.0297 & 1.4855 & 0.0297 & 0.02 & \\
\hline \multirow{3}{*}{$b / \mathrm{mm}$} & $1^{\text {st }}$ stage & 57 & 1.14 & 57 & 1.14 & 0.02 & \multirow{3}{*}{ normal } \\
\hline & $2^{\text {nd }}$ stage & 92 & 1.84 & 92 & 1.84 & 0.02 & \\
\hline & $3^{\text {rd }}$ stage & 114 & 2.28 & 114 & 2.28 & 0.02 & \\
\hline \multirow{3}{*}{$d / \mathrm{mm}$} & $1^{\text {st }}$ stage & 18 & 0.36 & 18 & 0.36 & 0.02 & \multirow{3}{*}{ normal } \\
\hline & $2^{\text {nd }}$ stage & 18 & 0.36 & 18 & 0.36 & 0.02 & \\
\hline & $3^{\text {rd }}$ stage & 22 & 0.44 & 22 & 0.44 & 0.02 & \\
\hline \multirow{3}{*}{$h / \mathrm{mm}$} & $1^{\text {st }}$ stage & 43 & 0.86 & 43 & 0.86 & 0.02 & \multirow{3}{*}{ normal } \\
\hline & $2^{\text {nd }}$ stage & 46.5 & 0.93 & 46.5 & 0.93 & 0.02 & \\
\hline & $3^{\text {rd }}$ stage & 57 & 1.14 & 57 & 1.14 & 0.02 & \\
\hline
\end{tabular}

According to the basic types and distribution parameters of variables in Table 2, the structural reliability and its sensitivity of gearbox are analyzed with accordance to the proposed SA-LS method based on the dichotomy of the golden section point, their results are summarized in Table 3 . It can be seen from Table 3 that the proposed algorithm can highly analyze the reliability and its sensitivity of the implicit nonlinear PF that is complicated and changeable in the non-normal variable space, its precision can be comparable with that of MCS, and its velocity is faster than that of MCS.
For the random variables whose MGF or CGF doesn't exist, we can't analyze the structural reliability and its sensitivity by applying the proposed method above until it is beforehand translated into the variable existing in CGF. For instance, for the random variable $x$ of the log-normal distribution in the example, it can be transformed into the normal variable $y$ by the formula $y=\ln x$, which is treated as a new variable $x$.We can thereby analyze the structural reliability and its sensitivity with accordance to the CGF of the normal distribution. 
Table 3. Results contrast of failure probability and its sensitivity by analyzing the optimal structure of eachstage gearbox with the present method and MCS, respectively

\begin{tabular}{|c|c|c|c|c|c|c|c|c|c|}
\hline \multirow[b]{2}{*}{ name } & \multicolumn{3}{|c|}{$1^{\text {st }}$ stage } & \multicolumn{3}{|c|}{$2^{\text {nd }}$ stage } & \multicolumn{3}{|c|}{$3^{\text {rd }}$ stage } \\
\hline & MCS & $\begin{array}{l}\text { present } \\
\text { method }\end{array}$ & $\begin{array}{l}\text { relative } \\
\text { error } / \%\end{array}$ & MCS & $\begin{array}{l}\text { present } \\
\text { method }\end{array}$ & $\begin{array}{l}\text { relative } \\
\text { error } / \%\end{array}$ & MCS & $\begin{array}{l}\text { present } \\
\text { method }\end{array}$ & $\begin{array}{l}\text { relative } \\
\text { error } / \%\end{array}$ \\
\hline$P_{f}$ & 0.0654 & 0.0663 & 1.32 & 0.0719 & 0.0715 & 0.56 & 0.0842 & 0.0833 & 1.07 \\
\hline $\begin{array}{c}\text { the number of } \\
\text { samples }\end{array}$ & $10^{6}$ & 2000 & - & $10^{6}$ & 2000 & - & $10^{6}$ & 2000 & - \\
\hline$\partial P_{f} / \partial \mu_{\sigma_{s}} \times 10^{-5}$ & -2.9483 & -2.9221 & 0.89 & -2.9682 & -3.0000 & 1.07 & -3.2391 & -3.2083 & 0.95 \\
\hline$\partial P_{f} / \partial \sigma_{\sigma_{s}} \times 10^{-5}$ & -4.2556 & -4.1892 & 1.56 & -4.2842 & -4.1921 & 2.15 & -4.6752 & -4.7748 & 2.20 \\
\hline$\partial P_{f} / \partial \mu_{S} \times 10^{-2}$ & -0.9768 & -0.9792 & 0.25 & -1.0181 & -1.0108 & 0.72 & -1.2313 & -1.2233 & 0.65 \\
\hline$\partial P_{f} / \partial \sigma_{S} \times 10^{-2}$ & .9049 & -2.8515 & 1.84 & -2.9894 & .9443 & 1.51 & -3.6701 & -3.6143 & 1.52 \\
\hline$\partial P_{f} / \partial \mu_{T_{s}} \times 10^{-6}$ & -5.9517 & -5.8106 & 2.37 & -1.5979 & -1.6385 & 2.54 & -0.4892 & -0.4806 & 1.76 \\
\hline$\partial P_{f} / \partial \sigma_{T_{s}} \times 10^{-5}$ & -1.3042 & -1.3440 & 3.05 & -0.3557 & 3667 & 3.08 & 0.1071 & -0.1051 & 1.83 \\
\hline$\partial P_{f} / \partial \mu_{k_{s}} \times 10^{-5}$ & -9.9452 & -9.7483 & 1.98 & -6.8935 & -6.7480 & 2.11 & -3.7696 & -3.8729 & 2.74 \\
\hline$\partial P_{f} / \partial \sigma_{k_{s}} \times 10^{-2}$ & -2.4491 & -2.3871 & 2.53 & -2.0308 & 2.0028 & 1.38 & -1.8132 & -1.8341 & 1.15 \\
\hline$\partial P_{f} / \partial \mu_{\varepsilon} \times 10^{-3}$ & 0144 & -1.0062 & 0.81 & 244 & 7087 & 2.17 & 32 & -0.2321 & 0.46 \\
\hline$\partial P_{f} / \partial \sigma_{\varepsilon}$ & -0.1057 & -0.1080 & 2.14 & -0.1120 & 0.1110 & 0.93 & -0.1247 & -0.1236 & 0.92 \\
\hline$\partial P_{f} / \partial \mu_{b} \times 10^{-5}$ & 3.3986 & 3.3310 & 1.99 & -0.6953 & -0.6801 & 2.19 & 0.3767 & 0.3858 & 2.35 \\
\hline$\partial P_{f} / \partial \sigma_{b} \times 10^{-3}$ & -2.9537 & -2.8943 & 2.01 & -1.8358 & -1.8591 & 1.27 & -1.6005 & -1.5783 & 1.39 \\
\hline$\partial P_{f} / \partial \mu_{d} \times 10^{-5}$ & 3.6385 & 3.5865 & 1.43 & 7.7353 & 7.4947 & 3.11 & -6.0084 & -0.5906 & 2.70 \\
\hline$\partial P_{f} / \partial \sigma_{d} \times 10^{-3}$ & -9.4087 & -9.5790 & 1.80 & -9.2537 & -9.1251 & 1.39 & -8.2639 & -8.1656 & 1.19 \\
\hline$\partial P_{f} / \partial \mu_{h} \times 10^{-5}$ & 7.2527 & 7.1265 & 1.74 & 2.4707 & 2.4324 & 1.55 & -3.1821 & -3.2492 & 2.11 \\
\hline$\partial P_{f} / \partial \sigma_{h} \times 10^{-3}$ & -3.9674 & -3.8758 & 2.31 & -3.6272 & -3.5858 & 1.14 & -3.2517 & -3.2917 & 1.23 \\
\hline
\end{tabular}

\section{Conclusion}

(1) Aiming at the structural reliability and its sensitivity of the implicit nonlinear $\mathrm{PF}$ that is complicated and changeable in the non-normal variable space, the paper proposes the SA-LS method by the dichotomy of the golden section point, which is built on the SA-LS method. The method not only fully inherits the merits of the LS method in the standardized normal variable space, but it effectively plays the traits that SA is of high precision when it is used to analyze the related reliability of the linear PF structure in the nonnormal variable space. What's more, it is no need of answering the derivative or partial one of the PF in such a way that it is very suitable to solve the implicit PF. Example shows that it can accurately estimate the reliability and its sensitivity of the nonlinear PF in the non-normal variable space.

(2) The limited MGF or CGF of the random variables result in a limited application of the SALS method based on the dichotomy of the golden section, therefore, for the random variables whose MGF or CGF doesn't exist. We can't analyze the structural reliability and its sensitivity by applying the proposed method until it is translated beforehand into the variable existing in CGF.

(3) The stress-strength interference model for gearbox is constructed by applying the principle of the stress-strength interference theory and the modified idea of the bending stress of the tooth root. This lays a foundation on building the theory model for the similar gearbox. 


\section{Acknowledgements}

This work was financially supported by the Foundation of Hunan Provincial Natural Science of China (Grant No.2015JJ2015), the Key Project of Science and Technology Program of Changsha, China (Grant No.ZD1601010), the Foundation of Hunan Educational Department (Grant No. 14C0094, 15C0122,15C0123), the Foundation of the talent introduction of Changsha University (Grant No. SF1204, SF1403), the Outstanding Youth Foundation of Hunan Educational Department (Grant No. 16B029) and the Foundation of Hunan Province Key Construction Disciplines of China (2016). Their support is gratefully acknowledged. We are also grateful to the reviewers whose comments were very helpful in the revision of this paper.

\section{References}

[1] Melchers R.E., Ahammed M., A fast approximate method for parameter sensitivity estimation in Monte Carlo structural reliability, Computers and Structures, 82(2004), 1, 55-61.

[2] Schueller G.I., Pradlwarter H.J., Koutsourelakis P.S., A critical appraisal of reliability estimation procedures for high dimensions, Probabilistic Engineering Mechanics, 19(2004), 4, 463-474.

[3] Pradlwartera H.J., Pellissetti M.F., Realistic and efficient reliability Methods estimation for aerospace structures, Computer Methods in Applied Mechanics and Engineering,194(2005), 12-16,1597-1617.

[4] Daniels H.E., Saddlepoint approximations in statistics, Ann Math Statist, 25(1954), 4,631650.

[5] Lugannani R., Rice S.O., Saddlepoint approximation for the distribution of the sum of independent random variables, Adv Appl Probab, 12(1980),2,475-490.
[6] Du X.P., Sudjianto A., First order saddle-point approximation for reliability analysis, AIAA J,42(2004),6,1199-1207.

[7] Song S.F., Lu Z.Z., The reliability sensitivity analysis based on saddlepoint approximation and its improved method, Chinese Journal of Theoretical and Applied Mechanics, 43(2011), $1,162-168$.

[8] Jin Y.J., Zhang Y.M., Zhang Y.L., Analysis of reliability and reliability sensitivity for machine components by Mean-value first order saddlepoint approximation, Journal of Mechanical Engineering, 45(2009),12, 102-107.

[9] Huang B.Q., Du X.P., Probabilistic uncertainty analysis by mean-value first order saddle point approximation, Reliability Engineering and System Safety, 93(2008), 2, 325-336.

[10] Amol M.D., Padmakar J.P., Tejinder P.S., Optimal sequence of hole-making operations using particle swarm optimization and shuffled frog leaping algorithm, Engineering Review, 36(2016),2,187-196.

[11] Fu Y.D.,Cheng X.Y.,Tang Y.H., Optimization Theory and Method, Beijing, National Defense Industry Press,2008.

[12] Abdelkhalak E. H., Seifedine K.,Global optimization method for design problems, Engineering Review, 36(2016),2,149-155.

[13] Gillespie C.S., Renshaw E., An improved saddlepoint approximation, Math Biosci, 208 (2007), 2,359-374.

[14] ZhangG.Q.,Gong X.S., Structural optimization for mechanical elements by modified immune genetic algorithm, Journal of Central South University(Science \& Technology),42 (2011), 11, 3359-3369.

[15] Zhang G.Q.,Gong X.S.,Wang H.H.,et al, Multi-objective optimization design on gear train of planetary reducer in shield tunneling machine based on reliably grey particle swarm optimization. Journal of Mechanical Engineering, 46 (2010),23,135-145.

[16] Long Z.Y., Mechanic Design, Beijing, China Machine Press, 2002. 